\title{
A Novel Model for Traffic Hurdle Detection with Quick VANET Cluster Updation Model
}

\author{
Needhi Lathar \\ Department of Information \\ Technology, CEC Landran
}

(Mohali), India

\author{
Shashi Bhushan \\ Department of Information \\ Technology, CEC Landran \\ (Mohali), India
}

\author{
Munish Mahajan \\ Department of Information \\ Technology, CEC Landran \\ (Mohali), India
}

\begin{abstract}
The vehicular ad-hoc networks are the networks made of vehicles in the movement. The vehicular movement management is the important task for the automatically driven vehicles, where the traffic management module must be capable of handling the traffic movement and track the traffic hurdles. In this paper, the primary focus has been kept on the traffic hurdles detection in the movement of the traffic in the VANET cluster. The proposed model is based upon the distance calculation and non-moving hurdle prediction. Afterwards the proposed model floods the information in the VANET cluster about the detected vehicular hurdle. The proposed model has been evaluated on the basis of transmission delay, network load and data loss. The experimental results have proved the efficiency of the proposed model in handling the network during the hurdle detection and updation situations.
\end{abstract}

\section{Keywords}

VANET, Hurdle Detection, Information Flooding, Traffic Movement Control.

\section{INTRODUCTION}

VANETs are formed by the vehicles which are equipped with the wireless devices capable of transmitting data. This is an advanced technology for Intelligent Transport Systems (ITS). The data which is transmitted to vehicles is hazardous related and the hazards can be road blocks, tree falls, boulders on road, snow pile up, landslide, road maintenance work etc. VANET provides 2 ways of communication: V2V (Vehicleto-Vehicle) and V2R (Vehicle-to-Road side unit). In V2V communication, like relay race, the information of any mishappening goes on passing via vehicle to vehicle. But in V2R communications, the Road Side Unit (RSU) act as the main transmitting body and aware the entire vehicles running in its transmitting range.

The transmitting of the data through nodal range depends on the mobility pattern of the nodes. Mobility pattern, as the name suggests, refers to the acceleration of the specific nodes. Mostly, a very high mobility is preferred for the transmission via nodes because with a very high mobility the data can be transmitted at an extremely faster pace and with a secured privacy. A specific mobility pattern can't be accessed as it is dependent on the conditions or situations prevailing on the road at that particular instant of time. Those situations can be like the speeding behaviour of the driver, whether it is fast or slow, the lane system on the road, traffic rules, urban or rural road driving and any natural hazard or mishappening occurring on the road. Thus, mobility pattern is not predictable.
Mobility of data faces few problems during its transmission. Some of them can be dissemination (non scattering of signals), traffic density in the range of nodes, aggregation, security and privacy reasons. The above mentioned problems are due to the attacks faced by mobile data. Various attacks are fabrication attack, alteration attack and replay attack. These kind of attacks are done by various attackers such as selfish driver, malicious attackers and pranksters. Many times the mobility also suffers attacks from insiders and outsiders. Insider acts as a mischievous node which might enter between two nodes to interrupt the transmission of data. This interruption causes the messages to be dropped in between. It also impersonates a node into another node by modifying the message. Outsider is a kind of intruder which attacks the data during the transmission aiming to misuse it. Attacks are classified into two broad categories. First one is physical attack which further occurs due to two problems tamper proof device and event data recorder and another attack is logical attack which occurs due to the virus, Trojan horse and protocol weak spot. After attaining high and proper mobility by nodes, still there exists a problem of collision of nodes due to various reasons. This collision leads to many security and privacy related threats which may cause a mess in a network of vehicles running on roads. Collisions of nodes take place because of some reasons. These may be loss of network connection in the range of data transmission. It also happens due to high traffic density on the road which may cause data bits to collide in a very absurd manner.

Collision problems in VANETs results in breaking of link between On Board unit (OBU) and Road Side Unit (RSU). OBU is a part of wireless device which deals with the connecting ability of two nodes. If data or information regarding any hazard on road does not pass via vehicle to vehicle due to collision then road accidents may be very prominently occurring in that particular range.

To deal with such a harmful aspect of collision, technology has provided many solutions also. There is a device named as TPD (Tamper Proof Device) whose job is to secure the information related to the vehicle like keys, driver's identity, trip detail, route, speed etc. This secret information suffers attacks from outsiders like pranksters. They do misuse with the data and make collision to occur on the road. So, it is very necessary to maintain a proper security control over TPD to avoid the havoc on the road. Secondly, when RSU broadcasts the nodal information then sometimes it may happen that the connectivity loses and the data might or not have been passed to some vehicles. So to avoid such confusions it is very important to maintain a record of the transferred data so that the vehicles who have not received the message might gain it the second time. For this the authorities are supposed to record the broadcasting history in a tabular form. 
Some advancements or improvements are required to be done in the existing two VANETs that is V2V and V2R. This would need a technology which would put together the working principles of both the ways of transmission of data. For this, one would need to link both V2V and V2R transmissions in a manner so that they can collectively work for broadcasting the message in the designed range. As V2V passes information via node to node and V2R needs a link between RSU and vehicles, so for the advancement RSU should be propagating signal to all the nodes in its range as well as each and every node would be receiving the same signal from RSU and passing it to the node which is not even in the range of RSU. The node which is passing the RSU's signal to the out of range node would be the one which lies nearby to the out of range node but still should be in the RSU range. If the last node ranging in the area of RSU somehow stops working then it is mandatory for the RSU that by inspecting the tabular record of its broadcasting, it immediately activates another node lying nearest to the out of range node to pass the message. This will enable a proper working of nodes and also create an awareness of newly happened hazard due to the non working of a specific node. The improvisation can also include an amendment in the existing range of RSU's so that the transmitted data can propagate in a much easier and continuous manner. This would be done by widening the range of RSU's to an appropriate and careful extent.

Hence, with the help of the existing VANET and the VANET produced after advancement, one can make any country and its roads safe and non-messy. A technologically excellent future on roads will be at extent.

\section{LITERATURE REVIEW}

Gadkari M. Y. et al. proposed a new algorithm namely Traffic Management with Density Aware Routing (TMDAR) which is based on realistic road topology. TMDAR exploits the road hierarchy information to transfer data from source to destination. The proposed protocol can be clustered into four important parts: 1) Route discovery using road hierarchy, 2) Greedy Forwarding, 3) Route selection using density information, 4) Route maintenance using periodic update. To conclude the results, the proposed TMDAR is tested against Ad Hoc On-demand Distance Vector (AODV) routing protocol which establishes a route to a destination only on demand. To evaluate the performance of the developed protocol, the routing metric was Average end-to-end delay and it was observed that TMDAR performs well under high mobility of VANET compared to AODV[18].

S. Shahid et al. proposed the performance of Dynamic MANET On-demand (DYMO) routing protocol which is successor to the dynamic source routing (DSR) and Ad Hoc On-demand Distance Vector (AODV) routing protocols. Internet engineering task Force (IETF) is developing DYMO. DYMO works much like the AODV routing protocol. The only and important difference between the two routing protocols is that the originator of the Route Request (RREQ) message using DYMO protocol will also get information about all intermediate nodes in the newly discovered path. In AODV, only information about destination node and the next hop is maintained, while in DYMO path to every other intermediate node is also known. It computes multiple paths that can improve routing performance. Due to traffic dispersion, it can perform load-balancing, minimize the energy consumed by nodes to prevent traffic congestion. It also supports Quality of Service (QoS) in terms of multiple metrics[14].
Yongquan CHEN et al. proposed a real time vehicle safety system. A prototype test platform, consisting of collision detection and classification module, vehicle-to-vehicle (V2V) communication module and graphic user interface (GUI) module, has been built for validating the performance. Collision detection requires the detection of all kinds of security levels so that at those levels, sensors for detecting collisions can be implemented. Some real-time vehicle status information could be obtained from standard On-Board Diagnostics (OBD) interface, such as the status of Electronic Control Unit (ECU). However, it is limited and only for diagnostics applications. If we would like to get more realtime operation parameters of the vehicle, we can add external sensors on car which helps in estimating collisions. GPS Based AODV (GBAODV) protocol for V2V communication is developed, which is based on vehicle-mounted GPS device. It relieves the load of the network (less no. of RREQs), reduces broken links and packet loss ratio, and shortens endto-end delay. GUI helps to advertise the driver about his/her destruction related information to other vehicles and integrating all these safety measures[15].

Ankita Dixit et al. proposed two enhanced AODV protocols Prior-AODV (P-AODV) and Improved AODV. P-AODV provides two ways to reduce control overhead. One way is restrict the number of route request packets and another way is to restricting route request based on distance. Improved AODV reduces the control overhead and provides route stability. In improved AODV routing protocol, a method with two step organization in route discovery and route selection process is proposed[19].

Vishal Kumar et al. primarily categorized various possible applications of vehicular network, along with its features, and implementation in the real world. Although, some characteristics of VANETs resembles with the characteristics of MANETs but there are specific features which can be categorized as follows: 1) High dynamic topology, 2) Frequent disconnected network, 3) Mobility modeling and Prediction, 4) Communication Environment, 5) Hard delay constraints, 6) Interaction with onboard sensors and, 7) Unlimited Battery Power and storage. Applications of VANETs are: 1) Safety oriented, 2) Commercial oriented, 3) Convenience oriented and, 4) Productive Application[16].

Asim Rasheed et al. proposed a Fleet/Convoy Management system which is based on the GeoNet architecture for VANETs. Fleet or Convoy management is a special case of special case of VANET where nodes require communication to same type of other nodes as well as to backend server. Critical applications have been identified which are required for improving the load management and load efficiency of such networks. The design is based on four major applications of location update, enabling vehicles to chat with each other using text and voice and providing emergency alarms through broadcast. The geographical locations of the nodes are continuously updated and displayed geographically on local dynamic maps. A test bed for the models has been developed, discussed and used for performance analysis[17].

M. A. Berlin et al. proposes a Selective Road Hazard Message Dissemination Protocol (SRHMDP) for delivering road hazard information to vehicles travelling on highways. This paper proposes the use of RSUs and V2V communication to selectively deliver hazard information to vehicles travelling towards the hazard location. Vehicles encountering the hazard will relay the information directly to the RSU which is within its communication vicinity. Otherwise, if there is no RSU available, it will forward the information to other vehicles to 
forward to the RSU preceding the hazard location. This would enable hazard information to be sent in a timely manner to vehicles travelling toward the hazard to enable them to take corrective action like re-routing and avoid travel delays and traffic congestion. The protocol includes procedures for verifying the authenticity of the messages by the RSUs[13].

\section{RESEARCH WORK REVIEW}

In this research, we have worked upon the node movement to avoid the hazardous routing scheme, VANET security issue of prankster attack. In this attack one to more VANET nodes propagates their false location and direction to other nodes in the cluster, which may cause accident or traffic jam. This attacking mechanism can be utilized by terrorist or selfish driver to mandate their intensions.

The proposed model is based upon the vehicular movement hurdles detection and information flooding in the whole VANET cluster. The proposed model has been designed in a way, where it detects the traffic hurdles produced due to vehicular node failures or collisions etc. The algorithm of the proposed model is described below:

1. Each node in the VANET cluster stores its location coordinates $(\mathrm{x}, \mathrm{y})$

2. When the node $\mathrm{X}$ receives the coordinates from neighbor nodes, it updates its runtime neighbor table with the coordinate information.

3. Node $\mathrm{X}$ then computes the distance of the node to evaluates the node distance according to its transmission radius.

$\operatorname{Res}=(X 1-Y 1)^{2}+(X 2-Y 2)^{2}$

If $\boldsymbol{R}^{2}<$ Res, Point is within the circle

If $R^{2}==$ Res, Point is on the boundary

If $R^{2}>$ Res, Point is outside the circle

Where,

$X 1, X 2$ are coordinates of target node

$Y 1, Y 2$ are coordinates of test node

$\boldsymbol{R}$ is transmission radius

4. Calculate the distance between node $\mathrm{X}$ and $\mathrm{Y}$ using formula:

Distance $=\sqrt{(X 1-Y 1)^{2}+(X 2-Y 2)^{2}}$

Where,

$X 1, X 2$ are coordinates of target node $\mathbf{Y 1}, \mathbf{Y 2}$ are coordinates of test node

5. Calculate the displacement of node $\mathrm{Y}$ using formula

$$
\begin{aligned}
& \text { Displacement }= \\
& \sqrt{(\boldsymbol{o Y 1}-\boldsymbol{n Y 1})^{2}+(\boldsymbol{o Y} 2-\boldsymbol{n Y} 2)^{2}} \\
& \text { Where, } \\
& \text { oY1, oY2 are old coordinates } \\
& n Y 1, n Y 2 \text { are new coordinates }
\end{aligned}
$$

6. Node X makes the decision logic and update other nodes in the cluster.

\section{RESULT ANLAYSIS}

The results from the simulation has been obtained in the form of various performance parameters which includes the End-toEnd delay, network load and packet loss. Packet loss increases the retransmissions and hence the network load increases which ultimately gives a rise to end to end delay. The proposed work has been entirely based upon the detection and updation of the hurdles in the vehicle paths which are responsible for flooding data and delivering wrong information. For the updates, the vehicles flood the data in the cluster, due to which the network load increases than the normal situations. The aim of this research is to lower the data volume during the detection and updation periods in comparison with the existing schemes. The proposed model has been designed with the communication efficiency, which controls the data volumes during the proposed work simulation. For a clear understanding of the proposed and existing schemes results are analyzed in both graphical and tabular forms.

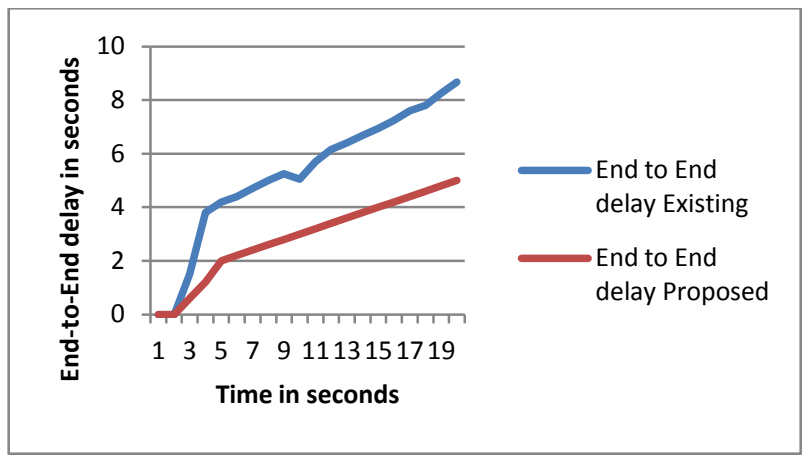

Figure: End-to-End delay

The End-to-End delay has been recorded on the lower limit as compared with the existing works, which is due to the communication efficiency of the proposed model. The end-toend delay indicates the transmission period of a packet. The end-to-end delay can be lowered using the efficient mechanisms for the data transmissions. The proposed work end-to-end delay can be considered efficient and performance booster. This slight improved scheme makes a huge change with performance which makes VANETs more efficient.

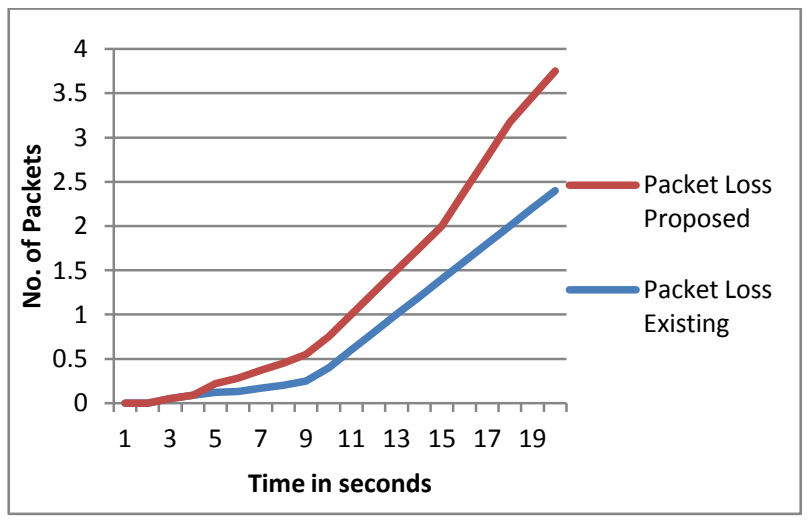

Figure 2: Packet Loss 
The packet loss is the indicator of data loss during the transmission due to the queue overflow, network load or other reasons. The lower is the packet loss the higher is the performance. The performance of the proposed work can be considered better in case of lower packet loss. The data loss has been observed lower in the proposed work. Lower packet loss helps to reduce the number of retransmissions and makes the network more efficient for the traffic without any delayed information.

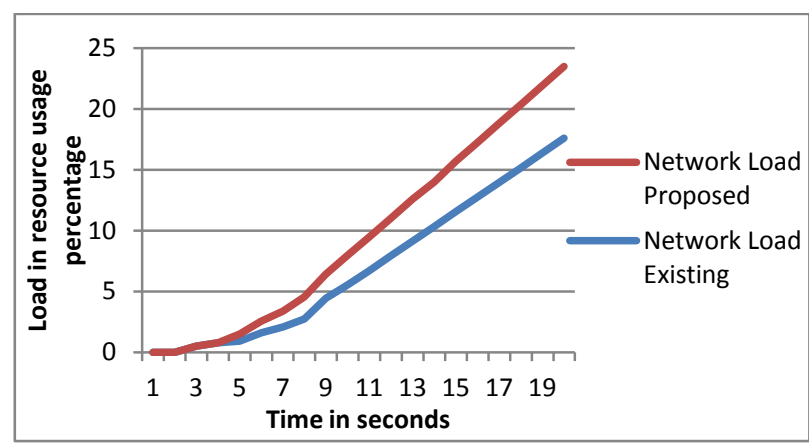

Figure 3: Network Load

The network load is the parameter, which indicates the performance of the network due to high volume traffic situations. The proposed work has been observed with the lower limit of the network load which indicates the enhanced performance of the proposed work.

The table given below contains comparison values between the existing and proposed schemes against the parameters of end to end delay, packet loss and network load:

\section{CONCLUSION AND FUTURE WORK}

The proposed model has been proposed for the detection and updation about the traffic hurdles in the urban traffic flow. The proposed work has been evaluated on the basis of end-toend delay or transmission delay, data loss in the form of packet loss and network load. The proposed work has been considered better than its counterparts. The proposed model has been evaluated under various situations and has performed better in all of the situations. The proposed model is considered efficient in terms of handling the traffic hurdles and information flooding in the cluster about the detected hurdles.

In the future, the proposed model can be enhanced for the higher order of hurdle detection with automatic alternative route controller, automatic movement rule deployment, traffic flow management or vehicular movement control.

\begin{tabular}{|c|c|c|c|c|c|c|}
\hline \multirow{2}{*}{$\begin{array}{l}\text { Sr. } \\
\text { No. }\end{array}$} & \multicolumn{2}{|c|}{ End to End Delay } & \multicolumn{2}{|c|}{ Packet Loss } & \multicolumn{2}{|c|}{ Network Load } \\
\hline & Existing & Proposed & Existing & Proposed & Existing & Proposed \\
\hline 1 & 0 & 0 & 0 & 0 & 0 & 0 \\
\hline 2 & 0 & 0 & 0 & 0 & 0 & 0 \\
\hline 3 & 1.5 & 0.6 & 0.05 & 0 & 0.5 & 0 \\
\hline 4 & 3.8 & 1.2 & 0.09 & 0 & 0.78 & 0 \\
\hline 5 & 4.2 & 2 & 0.12 & 0.1 & 0.91 & 0.6 \\
\hline 6 & 4.4 & 2.2 & 0.13 & 0.15 & 1.6 & 0.96 \\
\hline 7 & 4.7 & 2.4 & 0.17 & 0.2 & 2.1 & 1.3 \\
\hline 8 & 5 & 2.6 & 0.2 & 0.25 & 2.726667 & 1.83 \\
\hline 9 & 5.25 & 2.8 & 0.25 & 0.3 & 4.44 & 2 \\
\hline 10 & 5.05 & 3 & 0.4 & 0.35 & 5.56 & 2.41 \\
\hline 11 & 5.7 & 3.2 & 0.6 & 0.4 & 6.73 & 2.76 \\
\hline 12 & 6.15 & 3.4 & 0.8 & 0.45 & 7.939333 & 3.11 \\
\hline 13 & 6.4 & 3.6 & 1 & 0.5 & 9.148667 & 3.46 \\
\hline 14 & 6.68 & 3.8 & 1.2 & 0.55 & 10.358 & 3.65 \\
\hline 15 & 6.95 & 4 & 1.4 & 0.6 & 11.56733 & 4.16 \\
\hline 16 & 7.24 & 4.2 & 1.6 & 0.79 & 12.77667 & 4.51 \\
\hline 17 & 7.6 & 4.4 & 1.8 & 0.98 & 13.986 & 4.86 \\
\hline 18 & 7.8 & 4.6 & 2 & 1.17 & 15.19533 & 5.21 \\
\hline 19 & 8.25 & 4.8 & 2.2 & 1.26 & 16.40467 & 5.56 \\
\hline 20 & 8.67 & 5 & 2.4 & 1.35 & 17.614 & 5.91 \\
\hline 21 & 8.8 & 5.2 & 2.6 & 1.44 & 18.82333 & 6.26 \\
\hline
\end{tabular}




\section{REFERENCES}

[1] Ghaleb, Fuad A., M. A. Razzaque, and Ismail Fauzi Isnin. "Security and privacy enhancement in vanets using mobility pattern." In Ubiquitous and Future Networks (ICUFN), 2013 Fifth International Conference on, pp. 184-189. IEEE, 2013.

[2] Samara, Ghassan, Wafaa AH Al-Salihy, and R. Sures. "Security issues and challenges of vehicular ad hoc networks (VANET)." In New Trends in Information Science and Service Science (NISS), 2010 4th International Conference on, pp. 393-398. IEEE, 2010.

[3] Seuwou, Patrice, Dilip Patel, Dave Protheroe, and George Ubakanma. "Effective security as an ill-defined problem in vehicular ad hoc networks (VANETs)." In Road Transport Information and Control (RTIC 2012), IET and ITS Conference on, pp. 1-6. IET, 2012.

[4] Javed, Muhammad A., and Jamil Y. Khan. "A geocasting technique in an IEEE802. 11p based vehicular ad hoc network for road traffic management." InAustralasian Telecommunication Networks and Applications Conference (ATNAC), 2011, pp. 1-6. IEEE, 2011.

[5] Hung, Chia-Chen, Hope Chan, and EH-K. Wu. "Mobility pattern aware routing for heterogeneous vehicular networks." In Wireless Communications and Networking Conference, 2008. WCNC 2008. IEEE, pp. 2200-2205. IEEE, 2008.

[6] Dias, João A., João N. Isento, Vasco NGJ Soares, Farid Farahmand, and Joel JPC Rodrigues. "Testbed-based performance evaluation of routing protocols for vehicular delay-tolerant networks." In GLOBECOM Workshops (GC Wkshps), 2011 IEEE, pp. 51-55. IEEE, 2011.

[7] Moser, Steffen, Simon Eckert, and Frank Slomka. "An approach for the integration of smart antennas in the design and simulation of vehicular ad-hoc networks." In Future Generation Communication Technology (FGCT), 2012 International Conference on, pp. 36-41. IEEE, 2012

[8] Sumra, Irshad Ahmed, Halabi Hasbullah, J. A. Manan, Mohsan Iftikhar, Iftikhar Ahmad, and Mohammed Y. Aalsalem. "Trust levels in peer-to-peer (P2P) vehicular network." In ITS Telecommunications (ITST), 2011 11th International Conference on, pp. 708-714. IEEE, 2011.

[9] Sumra, Irshad Ahmed, Halabi Hasbullah, and J-L. A. Manan. "VANET security research and development ecosystem." In National Postgraduate Conference (NPC), 2011, pp. 1-4. IEEE, 2011.

[10] Chen, Lu, Hongbo Tang, and Junfei Wang. "Analysis of VANET security based on routing protocol information." In Intelligent Control and Information Processing (ICICIP), 2013 Fourth International Conference on, pp. 134-138. IEEE, 2013.

[11] Khabazian, Mehdi, and M. K. Mehmet Ali. "A performance modeling of vehicular ad hoc networks (VANETs)." In Wireless Communications and Networking Conference, 2007. WCNC 2007. IEEE, pp. 4177-4182. IEEE, 2007.

[12] Qian, Yi, Kejie Lu, and Nader Moayeri. "Performance evaluation of a secure MAC protocol for vehicular networks." In Military Communications Conference, 2008. MILCOM 2008. IEEE, pp. 1-6. IEEE, 2008.

[13] Berlin, M. A., and Sheila Anand. "Selective Road Hazard Message Dissemination Protocol for VANET." Asian Journal of Scientific Research 7, no. 4 (2014): 447.

[14] Shahid, S., N. U. Din, H. Zafar, K. Yahya, and L. Hasan. "Performance Evaluation of Routing Protocols in Vehicular Ad Hoc Networks." Pakistan Journal of Science 64, no. 1 (2012)

[15] Chen, Yongquan, Yuandong Sun, Ning Ding, Wing Kwong Chung, Huihuan Qian, Guoqing $\mathrm{Xu}$, and Yangsheng Xu. "A real-time vehicle safety system." In System Integration (SII), 2012 IEEE/SICE International Symposium on, pp. 957-962. IEEE, 2012.

[16] Kumar, Vishal, Shailendra Mishra, and Narottam Chand. "Applications of VANETs: Present \& Future." Communications and Network 5, no. 01 (2013): 12.

[17] Rasheed, Asim, Haleemah Zia, Farhan Hashmi, Umair Hadi, Warda Naim, and Sana Ajmal. "Fleet \& convoy management using VANET." Journal of Computer Networks 1, no. 1 (2013): 1-9.

[18] MY, Mr Gadkari. "Traffic Management on Road using Density Aware Routing." International Journal of Advanced Research in Computer Engineering \& Technology (IJARCET) 2, no. 4 (2013): pp-1530.

[19] Ankita Dixit, Shweta Singh, Kushal Gupta. "Comparative Study of P-AODC and Improved AODV in VANET." International Journal of Advanced Research in Computer Science and Management Studies(IJARCSMS) 3, no. 1 (2015). 